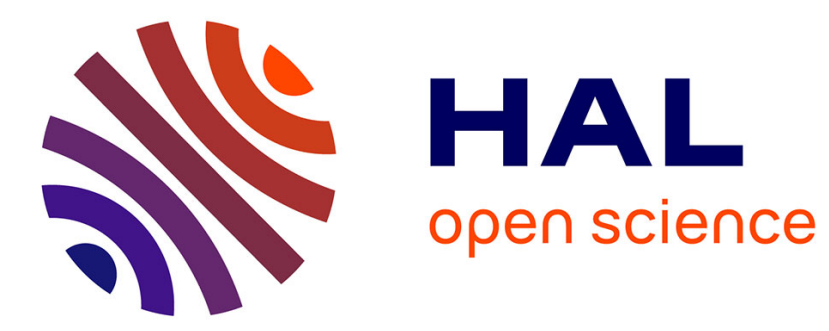

\title{
Performance improvement of a SOA-based coherent optical-OFDM transmission system via nonlinear companding transforms
}

\author{
Stéphane Azou, Serban Bejan, Pascal Morel, Ammar Sharaiha
}

\section{To cite this version:}

Stéphane Azou, Serban Bejan, Pascal Morel, Ammar Sharaiha. Performance improvement of a SOAbased coherent optical-OFDM transmission system via nonlinear companding transforms. Optics Communications, 2015, 336, pp.177 - 183. 10.1016/j.optcom.2014.09.067 . hal-01088835

\section{HAL Id: hal-01088835 \\ https://hal.science/hal-01088835}

Submitted on 28 Nov 2014

HAL is a multi-disciplinary open access archive for the deposit and dissemination of scientific research documents, whether they are published or not. The documents may come from teaching and research institutions in France or abroad, or from public or private research centers.
L'archive ouverte pluridisciplinaire HAL, est destinée au dépôt et à la diffusion de documents scientifiques de niveau recherche, publiés ou non, émanant des établissements d'enseignement et de recherche français ou étrangers, des laboratoires publics ou privés. 


\title{
Performance Improvement of a SOA-based Coherent Optical-OFDM Transmission System via Nonlinear Companding Transforms
}

\author{
Stéphane Azou ${ }^{1,2, *}$, Serban Bejan ${ }^{2,3}$, Pascal Morel ${ }^{1,2}$ and Ammar Sharaiha ${ }^{1,2}$ \\ ${ }^{1}$ IRT $b<>$ com, Brest, France \\ ${ }^{2}$ Ecole Nationale d'Ingénieurs de Brest (ENIB), Brest, France. \\ ${ }^{3}$ Military Technical Academy, Bucharest, Romania \\ *E-mail address: Stephane.AZOU@b-com.com; azou@enib.fr
}

\begin{abstract}
Coherent-Optical OFDM systems are known to be sensitive to large peak-to-average power ratio (PAPR) at the transmitter output, due to nonlinear properties of some components involved in the transmission link. In this paper, we investigate the impact of an amplification of such signals via a semiconductor optical amplifier (SOA), considering some recent experimental results. An efficient tradeoff between BER performance, computational complexity and power efficiency is performed by a proper design of Wang's nonlinear companding function, considered for the first time in an optical communication context. A BER advantage of around $3 \mathrm{~dB}$ can hence be obtained over a standard system implementation not using PAPR reduction. The designed function also proves to be more efficient than $\mu$-law function, considered in the literature as an efficient companding scheme.
\end{abstract}

Keywords: Optical fiber communication, CO-OFDM, Nonlinear predistortion, PAPR reduction, Semiconductor Optical Amplifiers.

\section{Introduction}

Orthogonal frequency-division multiplexing (OFDM) is considered as a promising technology for future fiberoptic communication networks [1] because of its many advantages, such as high spectral efficiency, simple compensation of linear channel impairments (chromatic and polarization mode dispersions), dynamic bandwidth allocation capability in a multiuser context (OFDMA), and powerful digital signal processing (DSP)-based implementation via fast Fourier transform (FFT) operations. Also, various system implementation variants are permitted, namely intensity-modulated with direct detection (IM/DD), coherent optical OFDM (CO-OFDM) and all-optical OFDM (AO-OFDM), so that the technology can be adopted both for long-haul optical transmission links [2] and metropolitan/access networks [3][4]. However, a well known drawback of OFDM signals is their high peak-to-average power ratio (PAPR) [5], which can give rise to distortions due to nonlinear properties of digital-to-analog converters (DAC), optical modulator, transmission medium or power amplifier (PA). Optical amplifiers are key components for most fiber communications systems and networks [6][7], due to their ability to compensate losses of the optical signal without requiring its conversion to the electric domain. Some broadband wavelength conversion capabilities have also been reported through use of semiconductor optical amplifiers (SOA) [8], provided that the incoming signal has low PAPR. It has been recently pointed out by Khaleghi et al. $[9,10]$ that SOA could be a pertinent choice for CO-OFDM signals amplification for their large optical bandwidth, small size and possibility of integration at limited cost [11]. Through numerical simulations and experimental investigations, the feasibility of SOA-based CO-OFDM transmission is demonstrated by the authors and the influence of some system parameters such as input power, signal wavelength and number of subcarriers is examined in terms of error-vector-magnitude (EVM). It is well known that for power efficiency considerations, the PA should be operated close to its saturation region, which can translates into large EVM, intersymbol interference (ISI) and significant out-of-band radiation if a SOA is used, due to its fast gain dynamics and nonlinear intrinsic properties. In order to improve the performances of the system studied by Khaleghi et al., we propose here to investigate some pre-distortion techniques for the multicarrier waveform in the electrical domain, with a main focus on peak power reduction. PAPR reduction has been an intensive research area over the last decade (see $[12,13]$ for recent surveys), due to the large number of subcarriers required for meeting high data rate and mobility demands. The many techniques identified in the literature can be broadly classified into signal distortion techniques, coding techniques and multiple signaling/probabilistic techniques. Most PAPR reduction methods have been investigated in a wireless communications context and relatively few references deal with the case of optical fiber communications. A short comparative study of a few methods, including Active 
Constellation Extension (ACE), precoding, selective mapping (SLM) and treillis shaping (TS), has been conducted by Goebel et al. [14]. More recently, a combination of SLM technique with optimized digital Mach-Zehnder (MZ) modulator pre-distortion has been proposed in [15]. Another approach is to use subcarrier pre-filtering [15] so as to reduce correlation between subcarriers. Hard clipping with predistortion was investigated in [17] and [18] to combat the nonlinearity in the MZ modulator, the nonlinear biasing and clipping effects being theoretically characterized in [19] for IM/DD optical OFDM systems. Biased clipping has also been proved to be beneficial for fiber nonlinearity mitigation in long-haul CO-OFDM systems [20]. Combining of data clipping and random additional phases has also been investigated in [21] in the context of OFDMA-based passive optical access networks. Hence, hard clipping (HC) has attracted significant attention, mainly due to its simplicity. Nevertheless, it causes additional noise (both in-band and out-of-band) that can degrade system performances, especially for high data rate and high modulation orders. This clipping noise can be mitigated via filtering but at the expense of an extra complexity. Companding (soft clipping) techniques appear to be more suitable for most of applications, for their ability to achieve excellent tradeoff between many factors that appear in the design of a PAPR algorithm: low computational complexity, no side information, good BER together with transparency to frame format, number of subcarriers, and constellation type. Such schemes rely on compressing large signals and enlarging small ones, in such a way that both PAPR reduction and immunity of small signals to noise is ensured. Until now, only $\mu$-law companding has been considered in the field of optical communications [22, 23, 24]. Since its introduction in [25], many other Nonlinear Companding Transforms (NCT) has been studied in the literature in order to avoid some drawbacks of $\mu$-law scheme, namely increase in the average power and lack of flexibility. In the present paper, we demonstrate that the SOA-based CO-OFDM system of Khaleghi et al. can be significantly improved by the use of an efficient NCT design. By considering a general scheme recently proposed by Wang et al. [26], we can achieve a precise control of the probability function of the OFDM signal through the use of a limited number of parameters. This is the first time this kind of promising approach is investigated for optical communications. Thanks to global optimization of some key parameters of the NCT algorithm, it is shown that a $3 \mathrm{~dB}$ advantage over a standard system implementation can be achieved in saturated regime. The good agreement of our results with the experimental data described in [10] proves the efficiency of the proposed setup and confirms that SOA could be a pertinent choice in a multicarrier optical transmission scenario.

\section{CO-OFDM system model}

In this study, we used a co-simulation setup of the CO-OFDM system which is similar to that used recently by Khalegi et al. [10], except that we implemented some blocks under Matlab rather than under VPItransmissionMaker for ease of companding algorithms implementation. The same SOA model is implemented using ADS software from Agilent Technology (the co-simulation involves ADS Ptolemy software); it relies on the carrier density rate and propagation equations of the optical signal field and the intensity of the Amplified Spontaneous Emission (ASE) noise. This model has been fitted to simulate a commercially available bulk $750 \mu \mathrm{m}$ long SOA (INPHENIXIPSAD1501), so that it yields a very good matching between simulated results and experimental results, as reported in [10]. The overall structure of the simulated system is illustrated in figure 1 ; it is very classical, except that we introduced a PAPR reduction block (the associated nonlinear companding will be explained in the next section).

The whole transmitter is implemented under Matlab, including laser diode (implemented according to [27]) and I/Q optical modulator (use of a standard nonlinear model as described in [28]). Our aim being to demonstrate the benefits of some PAPR reduction algorithms only, in presence of optical amplifier, we will assume in the following a linear electrical channel at IQ modulator input, a perfect coherent optical detector (ideal photodetectors), together with a perfect compensation of the laser phase noise on the receiver side; also influence of propagation over fiber is not considered. The ASE noise has been taken into account though, as companding algorithms can be affected by its non stationary nature. The noise field $\boldsymbol{E}_{A S E}(t)$ is computed according to

$$
\begin{gathered}
\boldsymbol{E}_{A S E}(t)=\sigma_{A S E}(t)\left[n_{1}(t)+j n_{2}(t)\right] \\
\sigma_{A S E}(t)=\sqrt{\frac{B_{N}}{2} P_{A S E}(t)}
\end{gathered}
$$

where $n_{i}(t), i=1,2$ denote independent Gaussian noise processes with zero mean and unit variance; $P_{A S E}(t)$ denotes the ASE power computed by the SOA model over an optical bandwidth of $\Delta \lambda=0.1 \mathrm{~nm}$ and the equivalent noise bandwidth per Hertz is expressed as

$$
B_{N}=\frac{B_{s i m} \lambda^{2}}{c \Delta \lambda}
$$




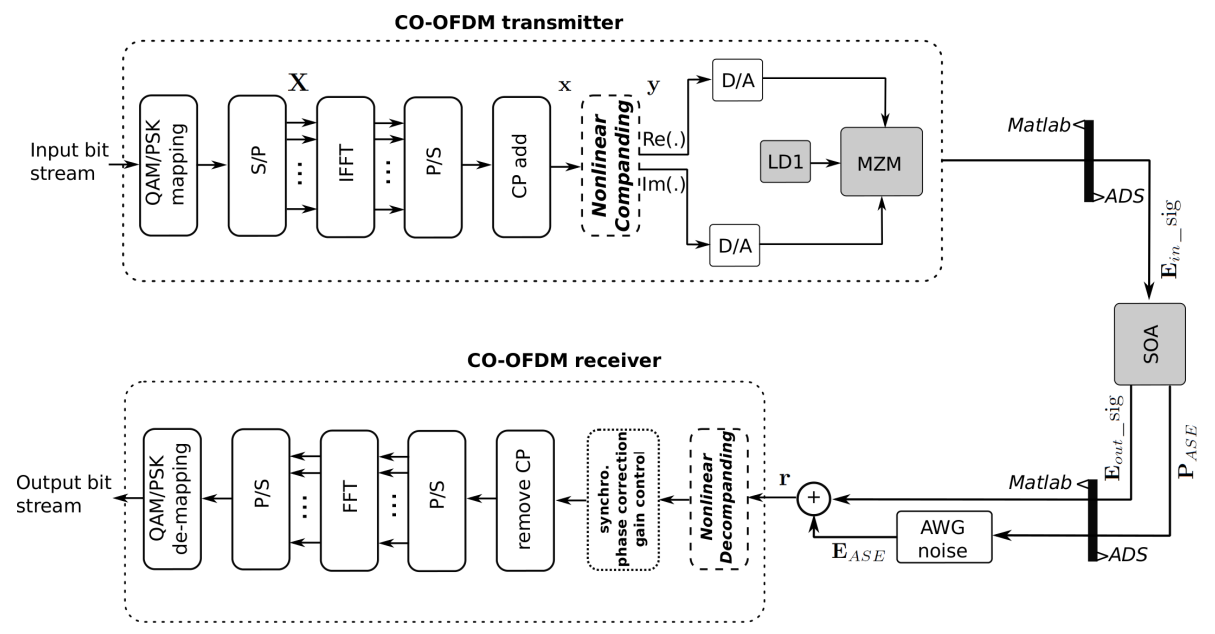

Figure 1: Block diagram of the CO-OFDM system with Matlab/ADS co-simulation, including nonlinear companding (S/P: serial to parallel; IFFT: inverse Fast Fourier Transform; CP: Cyclic Prefix; D/A: Digital to Analog conversion; AWG: Additive White Gaussian).

where $\lambda$ is the wavelength at which the simulation is performed, $c$ is the speed of light and $B_{\text {sim }}$ stands for the total simulation bandwidth.

The blocks implemented on the receiver side, not detailed in figure 1, are the same as in a standard CO-OFDM system, except that we perform inverse companding first once the signal field $\boldsymbol{r}(t)=\boldsymbol{E}_{\text {out }} \operatorname{sig}(t)+\boldsymbol{E}_{A S E}(t)$ has been coherently detected and pre-processed. The pre-processing consists in the following steps : the amplifier insertion losses are taken into account, a synchronization is performed thanks to first pilot symbols inserted in preamble of transmitted frame, phase shift due to SOA is corrected and power is adjusted.

\section{NCT optimal design}

The general idea behind the concept of nonlinear companding is to predistort the signal amplitude at the transmitter side so that the received signal is less affected by the nonlinear characteristics of some components of the communication link (a main focus is on high power amplifiers due to saturation phenomena). Hence, the original signal samples $x_{n}$ have their amplitude modified according to a particular nonlinear function $h$, the resulting signal $y_{n}=h\left(x_{n}\right)$ then being converted into analog waveform (see Fig. 1). At the receiver side, the received noisy signal $r_{n}=y_{n}+v_{n}$ is then transformed by the de-companding function in order to recover original signal (plus noise) : $\widetilde{x}_{n}=h^{-1}\left(r_{n}\right)=x_{n}+h^{-1}\left(v_{n}\right)$. Designing a good companding function is not a trivial task and depending on the underlying application, many criteria are sometimes to be considered (PAPR reduction, BER, bandwidth efficiency, complexity,...). Just enlarging small amplitude samples and compressing large amplitude samples in a blind manner can lead to poor system performances. The $\mu$-law companding belong to first generation of companding methods, having a main focus on the shape of the function $h($.$) . Despite the fact that such approaches can work satisfactorily$ in certain applications, including optical fiber applications as already mentioned, they offer poor flexibility for achieving balanced system performances. Then, other techniques have been introduced in the literature with the aim of shaping the statistics of the companded signal. In this paper, we consider the recent scheme of Wang et al. [26], which enables to construct a function $h($.$) so that the companded signal amplitude matches a target PDF$ $f_{\left|y_{n}\right|}(x)$ expressed as a piecewise linear function. This function is composed by two parts with distinct slopes $k_{1}>0$, $k_{2}<0$, with an inflexion point located at $c A(0<c<1)$ and a cutoff point at $A>0$. Only the two key parameters $\left\{k_{2}, c\right\}$ need to be chosen in practice, as the remaining coefficients can be deduced thanks to PDF definition and constant average power level property :

$$
\left\{\begin{array}{c}
k_{1}=\frac{2-A^{2} k_{2}(c-1)^{2}}{A^{2} c(2-c)} \\
A=\left(\frac{1}{2 \zeta_{2}}\left(\left(\zeta_{1}^{2}-4 \zeta_{0} \zeta_{2}\right)^{\frac{1}{2}}-\zeta_{1}\right)\right)^{\frac{1}{2}}
\end{array}\right.
$$

where $\zeta_{0}=12 \sigma^{2}(c-2), \zeta_{1}=-2\left(c^{3}-4\right), \zeta_{2}=k_{2}\left(c^{3}-3 c+2\right)$ and $\sigma^{2}=E\left\{\left|x_{n}\right|^{2}\right\}$.

The companding function resulting from this PDF design can then be expressed as 


$$
h(x)=\left\{\begin{array}{c}
\operatorname{sgn}(x) \sqrt{\frac{2}{k_{1}}\left(1-e^{-\frac{|x|^{2}}{\sigma^{2}}}\right)},|x| \leq \chi_{0} \\
\operatorname{sgn}(x) \frac{1}{k_{2}}\left(\left(k_{2}-k_{1}\right) c A+\sqrt{\left(k_{1}-k_{2}\right) k_{1} c^{2} A^{2}+2 k_{2}\left(1-e^{-\frac{|x|^{2}}{\sigma^{2}}}\right)}\right),|x|>\chi_{0}
\end{array}\right.
$$

where $\chi_{0}=\sigma\left(-\ln \left(1-\left(\frac{k_{1}}{2} c^{2} A^{2}\right)\right)\right)^{1 / 2}$.

The corresponding de-companding function takes the following form :

$$
h^{-1}(x)=\left\{\begin{array}{c}
\operatorname{sgn}(x) \sigma \sqrt{-\ln \left(1-\frac{k_{1}}{2}|x|^{2}\right)},|x| \leq c A \\
\operatorname{sgn}(x) \sigma \sqrt{-\ln \left(-\frac{k_{2}}{2}|x|^{2}+\left(k_{2}-k_{1}\right) c A|x|+1-\frac{c^{2} A^{2}}{2}\left(k_{2}-k_{1}\right)\right)}, \quad|x|>c A
\end{array}\right.
$$

The companding/de-companding processings do not require large computational cost, especially due to the fact that the operations can be performed by pre-computing numerical values of the functions and use of look-up tables. This makes NCT an attractive approach for high speed optical communications.

Until now, the Wang's NCT (WNCT) has only been investigated theoretically and no result is reported regarding its application for optical OFDM transmissions. Does this general scheme really bring something new for improving a system such as that recently studied by Khaleghi? How much performance can be gained from its use, with respect to a standard clipping scheme?

To answer these questions, we proceeded to global optimization with the objective of minimizing EVM. Due to the potentially complex cost surface of the corresponding function $f_{E V M}(\boldsymbol{p})$ for different values of the parameter vector $\boldsymbol{p}=\left[c, k_{2}\right]$, we used a genetic algorithm ${ }^{1}$ to solve the problem. Based on the principles of genetics and natural selection, such algorithms are known to be powerful for optimizing difficult problems [29], due to their ability to jump out of local minima. Our aim is to find

$$
\boldsymbol{p}^{*}=\arg \min _{\boldsymbol{p} \in \mathcal{D}} f_{E V M}(\boldsymbol{p})
$$

where the search space has been chosen as $\mathcal{D}=\left\{\left(c, k_{2}\right) / 0.001<c<0.5,-0.5<k_{2}<-0.001\right\}$; these restrictions for the search intervals were motivated by speeding up convergence of the algorithm, taking into consideration that $k_{2} \simeq-0.5$ or $c \simeq 0$ means very small $\mathrm{PAPR}$ reduction, whereas $k_{2} \simeq 0$ or $c \simeq 0.5$ yields large PAPR reduction. Also, due to huge computational time requested by the ADS model of the SOA, a population size of 10 has been specified, with 20 generations. These values may be judged to be too small at first, but as we will see in the sequel it suffices for achieving a significant performance improvement. Note that use of a hybrid function is a good way to compensate the small population size (it consists in running an additional optimization step after the genetic algorithm terminates). A total number of function evaluations of 261 has been performed, with the following OFDM parameters: frame size of $2^{11}$ QPSK modulated symbols, 128 subcarriers and a useful symbol duration of $22.5 \mathrm{~ns}$ with guard interval length of $1 / 8$, which means a rate close to $10 \mathrm{~Gb} / \mathrm{s}$. A peak voltage of $1 \mathrm{~V}$ at IQ modulator input has been considered so as to avoid any significant nonlinear effect for this stage (some investigations regarding robustness of Wang's companding scheme to any uncertainty of the peak voltage value will be conducted in the sequel). The input power of the SOA, biased at $200 \mathrm{~mA}$, was around $-16 \mathrm{dBm}$, so that the amplifier operates slightly above its saturation point. The solution resulting from the minimization process was found to be $c^{*}=0.43$, $k_{2}^{*}=-0.25$. As will be seen later (section 4), optimum parameters should be recomputed when the input power is changed (in the saturated region) and a simple look-up-table could then be used to automatically adapt $\left\{c, k_{2}\right\}$ with respect to the chosen operating point.

Optimized companding and de-companding functions at the chosen operating point are illustrated in Fig. 2.a. The simulated and target PDFs of the OFDM signal after optimal companding are depicted in Fig. 2.b; for comparison purposes, the PDF resulting from $\mu$-law companding transform with $\mu=2$ is illustrated on the same figure ( $\mu=2$ was also found to be the best companding coefficient in [24]). It can be observed that both methods give almost the same average magnitude after companding. In case of $\mu$-law companding, we can observe that the PDF has a similar shape than the original one, but with a compression of the original signal portions having small or large amplitudes (below 0.1 and above 0.25 ); in the medium range, a slight expansion is noted. As expected, NCT yields a very different PDF; it can be noticed that all the peaks exceeding $A \simeq 0.245$ have been removed and that the $\mathrm{PDF}$ is almost constant above the inflexion point $c . A \simeq 0.1$, which is very close to the value maximizing

\footnotetext{
${ }^{1}$ Functions ga.m and gaoptimset.m embedded in the Global Optimization toolbox under MatLAB (The MathWorks, Inc.).
} 
the original PDF. One more observation is that both PDFs after companding are almost the same over the range $[0, c A]$.

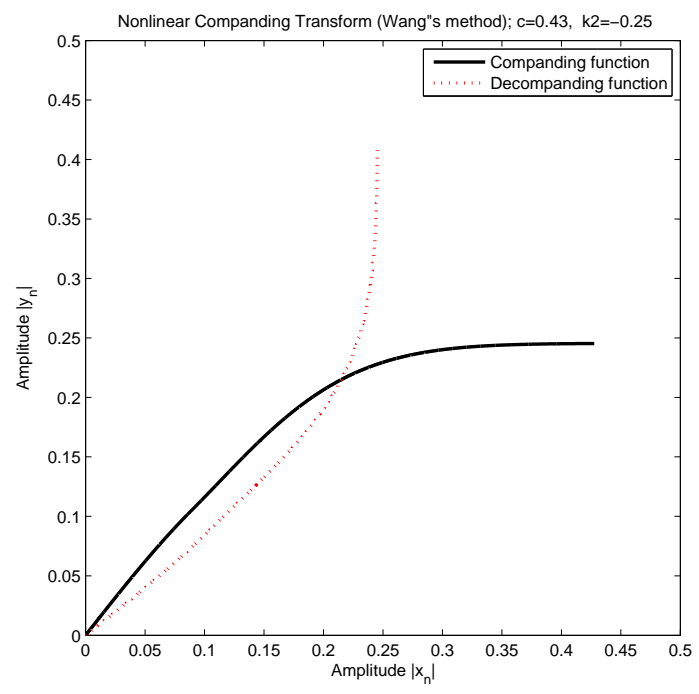

(a)

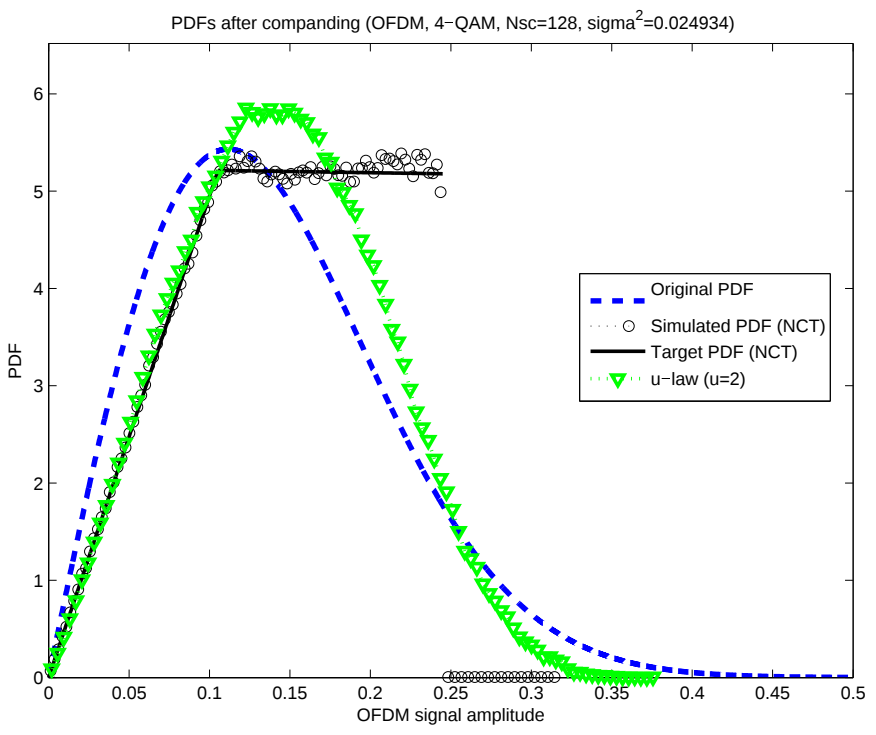

(b)

Figure 2: (a) Optimal companding and de-companding functions $\left(c^{*}=0.43, k_{2}^{*}=-0.25\right)$; (b) Original and simulated/theoretical PDFs of the OFDM signal after companding.

\section{Numerical results}

Many numerical simulations were performed to evaluate the overall performance improvement resulting from optimum WNCT design. First of all, we checked the good agreement between the results derived via our cosimulation software and the experimental data obtained by Khaleghi et al.. To do that, we considered a $10 \mathrm{~Gb} / \mathrm{s}$ CO-OFDM transmission with a bit stream made of $2^{11}$ QPSK symbols spread over 128 subcarriers, amplified by a SOA booster biased at $200 \mathrm{~mA}$. Before entering optical modulator, the signal was hard-clipped with a ratio of 6 $\mathrm{dB}$, as in [10]. The EVM has been computed for different values of the amplifier input power, considering an ideal coherent detection of the output signal. Because some imperfections of the transmission chain have not been taken into account in our simulations, the EVM curve obtained was not in perfect agreement with experimental data at first, but a very good correlation was noticed. After some fitting between experimental and simulated results, consisting in a shifting of $+3.4 \mathrm{dBm}$ for the input power and of $+3 \%$ for the EVM, a very good matching was achieved. These corrections have been applied to all PAPR reduction schemes we studied so as to reflect the EVM that can be expected in practice. For distortion-based techniques, we considered in particular: hard-clipping with a clipping ratio of $5 \mathrm{~dB}$ or $6 \mathrm{~dB}, \mu$-law companding with a parameter $\mu$ equal to 1 or 2 , and optimized WNCT. The classical Selective Mapping (SLM) approach, which has been pointed out as being effective for CO-OFDM systems in [14][15], has also been examined by multiplying original data block by 16 random phase sequences with phase values taken in $\{-1,+1,-j,+j\}$ prior to IFFT. Note that, contrary to distortion-based methods, the latter approach reduces transmission rate because $\left\lfloor\log _{2} M\right\rfloor$ bits (with $M=16$ here) need to be transmitted as side information to the receiver to allow recovery of the original data.

The Complementary Cumulative Distribution Function (CCDF) has been computed first, in order to measure the ability of various algorithms to reduce PAPR; the results are depicted in Fig. 3. As can be seen, the reduction is rather weak with use of $\mu$-law method or SLM (note however that results depend on the value chosen for $M$ ). Probability of large amplitudes is largely decreased thanks to HC, but at the expense of few BER improvement, as will be seen in the following. WNCT clearly yields the maximum PAPR reduction in our simulation setup; a difference of around $7 \mathrm{~dB}$ is noticed with respect to original OFDM signal at a target probability of $10^{-3}$. 


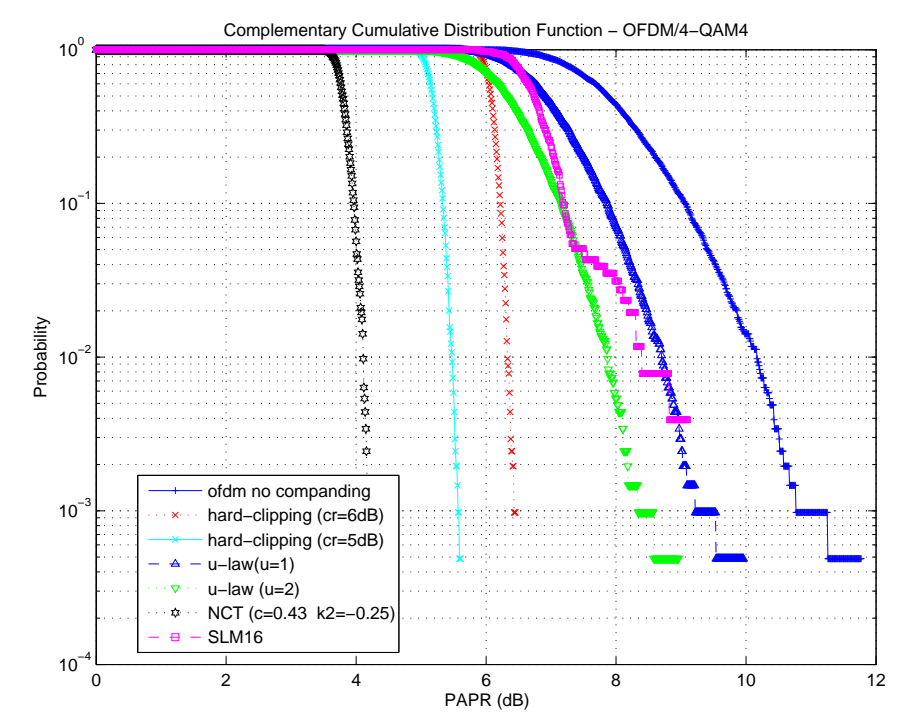

Figure 3: Comparison of CCDF for various PAPR reduction schemes.

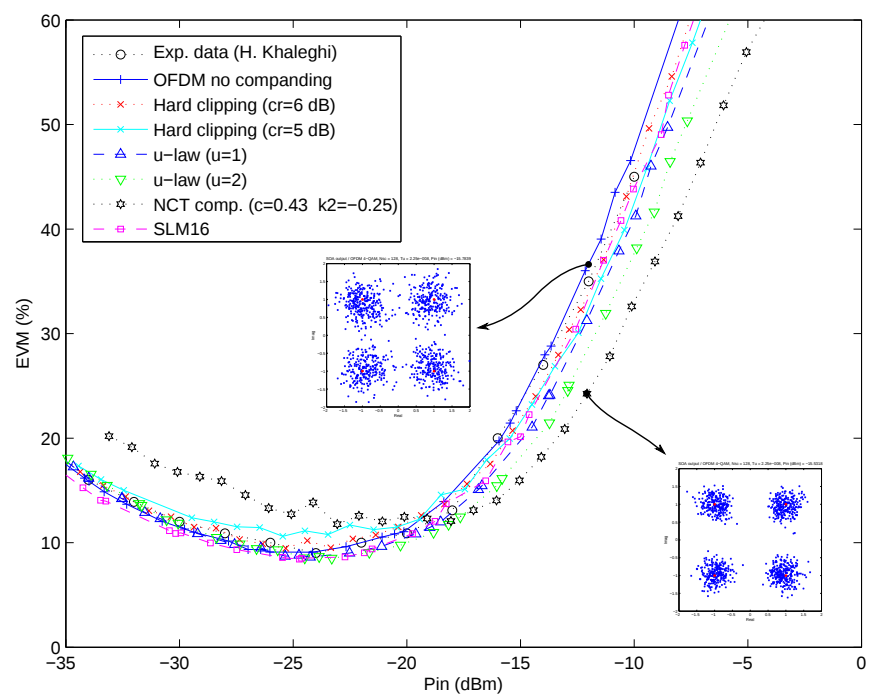

Figure 4: Simulated EVM using co-simulation setup as a function of SOA input power of different PAPR reduction techniques; the inserts show the corresponding received constellations for original CO-OFDM system (with no PAPR reduction) and WNCT-based system.

Figure 4-5 show the EVM performance of various algorithms against SOA input power. First, the good fitting between Khaleghi's experimental results and simulations for $6 \mathrm{~dB} \mathrm{HC}$ can be observed. Very close results are obtained if $5 \mathrm{~dB} \mathrm{HC}$ is used; only a slight improvement can be seen in this case for strongly saturated regime, $6 \mathrm{~dB}$ $\mathrm{HC}$ being slightly better in linear regime. What can also be observed is that PAPR reduction is required once the amplifier operates in the saturation region, due to rapid degradation of EVM as a result of large peaks of amplitude; inversely, there is no need to use PAPR reduction if the amplifier is not saturated. Nonetheless, it can be seen that SLM is the only approach enabling a slight improvement in this case. $\mu$-law method performs favorably over a wide range of input power, especially for $\mu=2$; an improvement of around $2 \mathrm{~dB}$ is noticed with respect to original CO-OFDM signal for an EVM of 15\%. As expected, optimized WNCT appears to be the most efficient approach for input power exceeding -17 dBm. Recall that the companding/decompanding functions have been optimized for an operating point located at $-16 \mathrm{dBm}$. Consequently, a rapid performance degradation occurs when the input power is below this threshold and sensitivity of WNCT to ASE noise is observed (this point will be investigated in the sequel). From a practical point of view, it would be actually pertinent to compute optimal parameters $\left\{c, k_{2}\right\}$ for various input power values (from $-20 \mathrm{dBm}$ to $-10 \mathrm{dBm}$, for example) and using a look-up-table so as to get best EVM whatever the input power is (adaptive WNCT). From the results obtained, we can also suggest a combining 

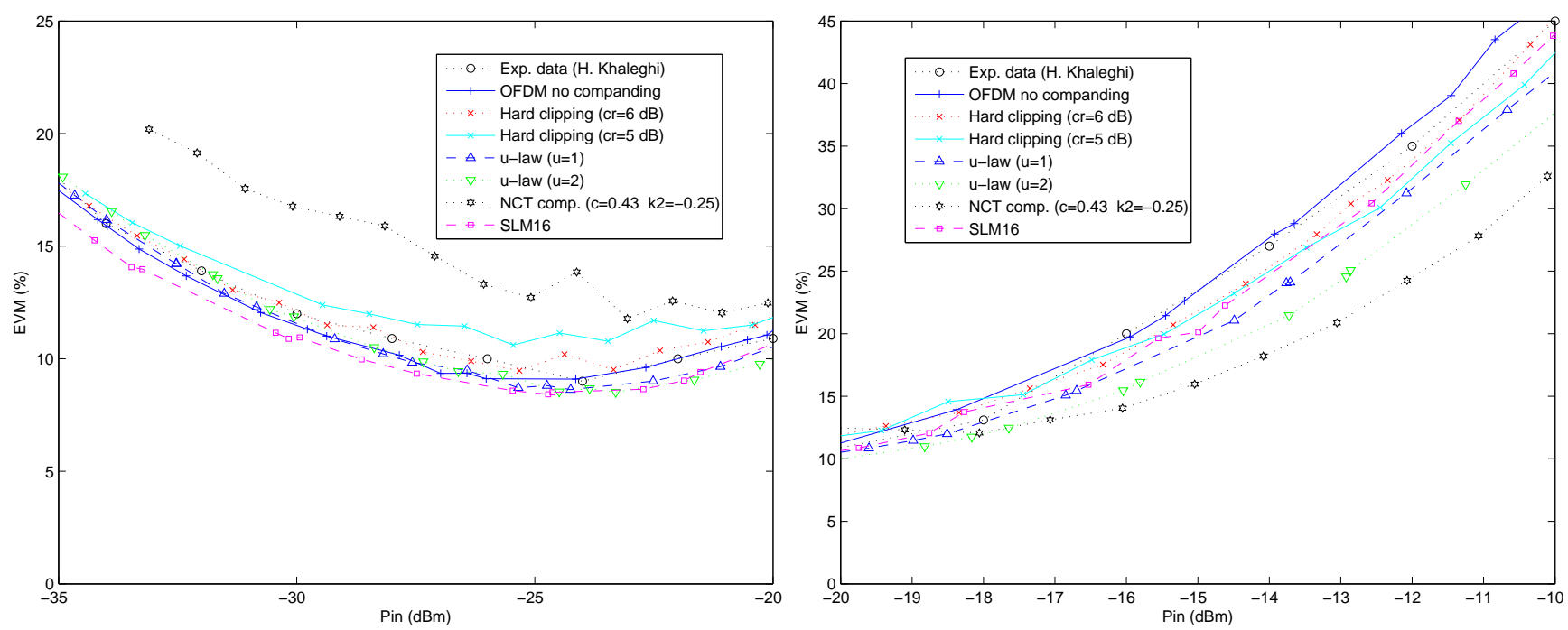

Figure 5: EVM vs. input power for differenct PAPR reduction techniques; Closeup views. (a) linear regime, (b) saturated regime.

of $\mu$-law and WNCT to improve system performances over a wide operating range.

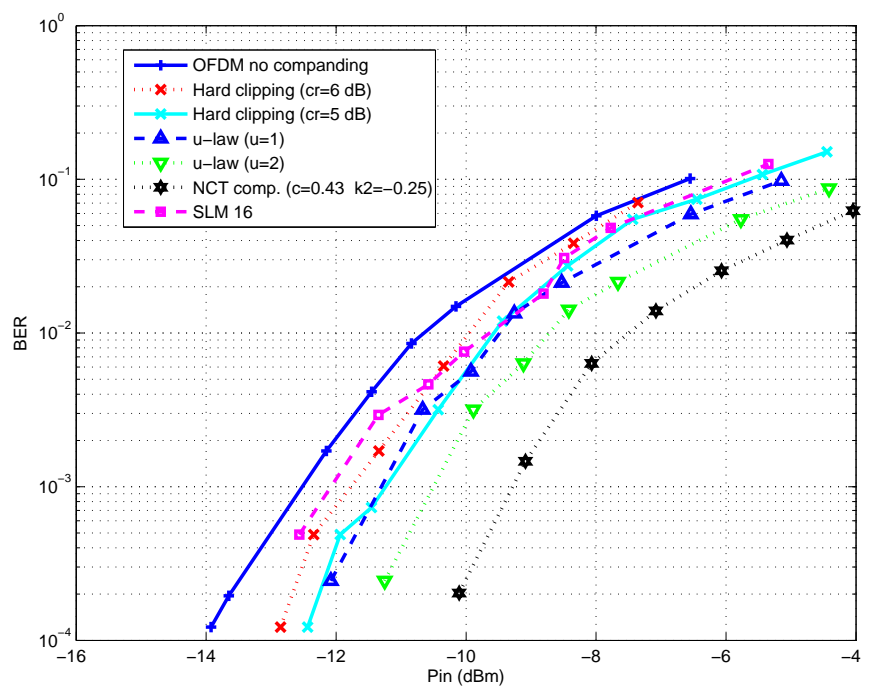

Figure 6: BER performance of various PAPR reduction methods for CO-OFDM with amplification via SOA.

The BER vs. input power has also been evaluated for the various methods (Fig. 5). Similar conclusions can be drawn when the amplifier is operated above the saturation point, that is WNCT clearly offers the best improvement: advantage of $3 \mathrm{~dB}$ over original OFDM signal at a target BER of $10^{-4}$, and advantage of around $1 \mathrm{~dB}$ over $\mu$-law method. If we take into consideration the fact that WNCT and $\mu$-law algorithms require similar (limited) computational costs, and that WNCT does not translate into power increase, this later approach clearly is the best compromise for improving system performances (including power efficiency). High flexibility in designing companding function is another key advantage of this approach.

As already mentioned, one drawback of WNCT is its sensitivity to ASE, which has been evaluated by excluding ASE in additional simulations. The results are illustrated by figure 7. From [10], we already know how ASE noise influences EVM performances of the original CO-OFDM system (with no use of PAPR reduction); below the compression point of the SOA (situated near $-25 \mathrm{dBm}$ ), a rapid degradation of EVM is observed as the input power decreases, due to increasing noise power. When the operating point enters the saturated regime (above $-25 \mathrm{dBm}$ ), it can be seen that ASE noise effect becomes negligible (almost the same EVM is noticed for original CO-OFDM system with or without ASE), the rapid performance degradation being mainly caused by various nonlinear effects of the component (FWM, SPM, SGM). The system behaves similarly if WNCT-based PAPR reduction is employed: 
the same EVM performance is observed above $-16 \mathrm{dBm}$ with or without noise; and as soon as some ASE noise appears performance is impacted. As a result of the decompanding function shape (see Fig. 2), a small change in signal amplitude due to noise around peaks can translate into significant distortion after decompanding. But, as already denoted, noise sensitivity is not really a limiting factor of WNCT because PAPR reduction is usually not used in case of linear regime.

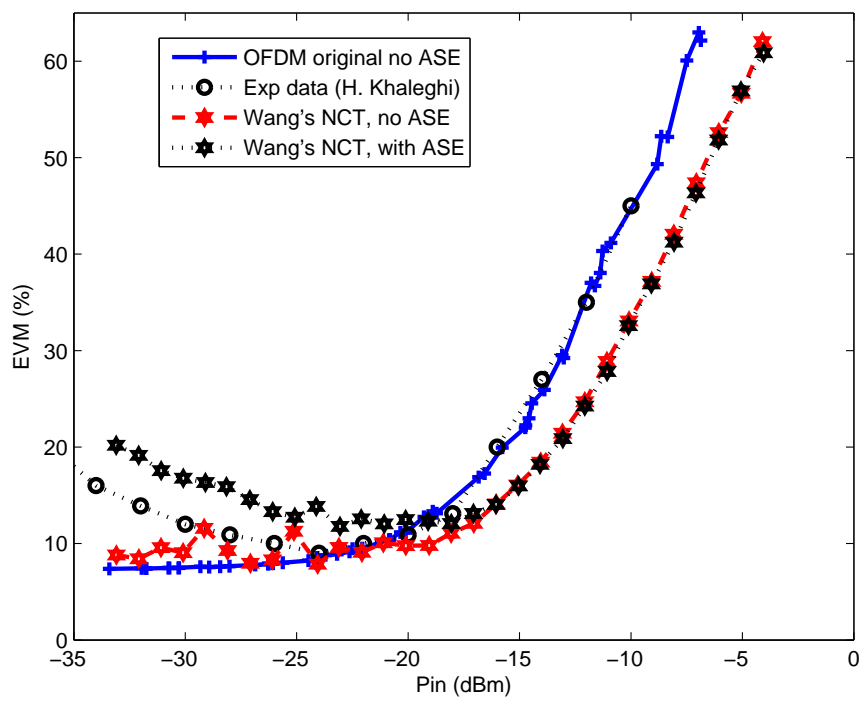

Figure 7: Impact of ASE noise over WNCT

As pointed out in some papers (e.g. [22, 17]), the resolution of analog-to-digital converter (ADC) can have a significant impact on the overall CO-OFDM system performances. If a distortion-based PAPR reduction is applied, it is even more critical to investigate the influence of the quantization step. Additional simulations have then be conducted for 4-bits and 8-bits resolutions, the results being depicted in figure 8. With no surprise, a low ADC resolution (4-bits) combined with ASE noise leads to poor EVM performances if WNCT is used; in this case, a benefit is obtained only in highly saturated regime (input power above $-14 \mathrm{dBm}$ ). For more realistic values of ADC resolution (8-bits), WNCT scheme proves to be rather robust and offers a net gain in performance.

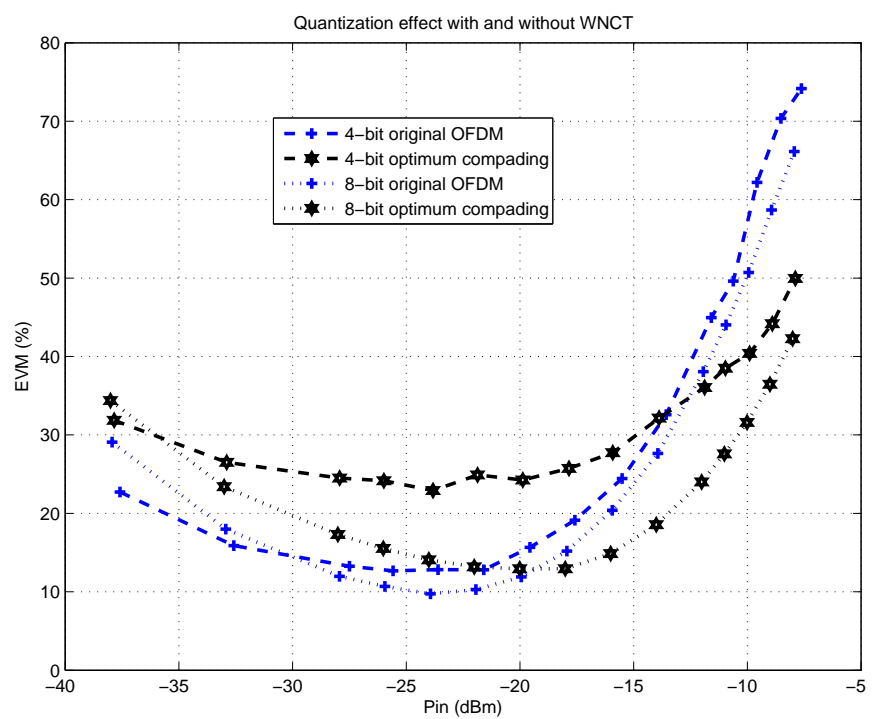

Figure 8: EVM performance of 4- and 8-bits quantization with and without companding for CO-OFDM with amplification via SOA

So far, a precise knowledge of the channel characteristics has been assumed, which could be viewed as a limitation in practice as it can be hard to maintain the operating conditions of the system components at prescribed values over time. As mentioned earlier, only one particular operating point has been considered for designing Wang's 
companding function. It is thus important to check that the approach still performs favorably in presence of any channel uncertainty. We investigated this issue by changing signal power at the IQ modulator input while keeping the same companding parameters $\left(c=0.43, k_{2}=-0.25\right.$.), the objective being to check if WNCT is still effective when a significant change regarding system settings occurs. Fig. 9 compares the EVM performances for different values of the peak voltage $V_{p}$ at optical modulator input. The initial simulations were accomplished for $V_{p}=1 \mathrm{~V}$ so as to avoid any significant modulator nonlinearity. A shift in voltage amplitude leads to almost no EVM variation as far as we stay in the linear region of the IQ modulator transfer function. A significant change in signal dynamics is noticed only for $V_{p} \gtrsim 3 \mathrm{~V}$, that is for large difference with initial value. As can be seen on the figure, a large uncertainty in $V_{p}$ (we considered $V_{p}=3 \mathrm{~V}$ and $V_{p}=4 \mathrm{~V}$ ) translates into an EVM degradation in a first part of the amplifier's saturation region (up to $P_{\text {in }} \simeq-11 \mathrm{dBm}$ for $V_{p}=3 \mathrm{~V}$; up to $P_{\text {in }} \simeq-10 \mathrm{dBm}$ for $V_{p}=4 \mathrm{~V}$ ) and a slight EVM improvement for high saturation. From these first investigations, we can see that WNCT seems quite robust to channel uncertainties. However, even if the performances are still acceptable in terms of BER $\left(<10^{-3}\right)$, a re-optimization of the predistortion function would be more suitable in such conditions.

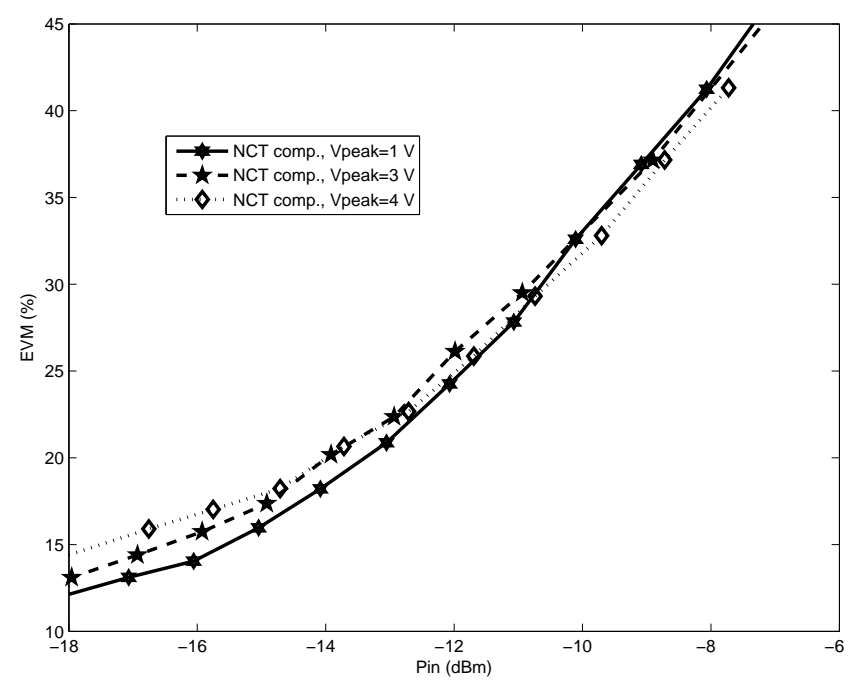

Figure 9: Influence of voltage amplitude at IQ modulator input on the EVM performance with WNCT.

\section{Conclusion}

The design of PAPR reduction algorithm has been investigated in this paper so as to improve the performance of CO-OFDM transmissions amplified by SOA. We particularly focused on some nonlinear companding schemes which can enable a good tradeoff between BER reduction and implementation complexity. An efficient design of Wang's nonlinear companding transform has been proposed; as a result, a $3 \mathrm{~dB}$ BER improvement is achieved when the amplifier is used in saturated regime. A key advantage of the approach is that a broad set of predistortion functions can be generated with only two parameters, so that the PAPR and more generally the dynamics of the transmitted signal is controlled. In this paper we considered only one operating point for optimizing WNCT, but the implementation could be easily extended by using a look-up-table containing various companding functions optimized for different power values at SOA input. These results confirm the pertinence of using SOA for CO-OFDM signals amplification and enable a more favorable system power efficiency.

Acknowledgment. This work has been supported by the IRT B-Com (http://www.b-com.com). The authors would like to thank the anonymous reviewers for their valuable comments and suggestions to improve the quality of the paper.

[1] W. Shieh and I. B. Djordjevic, OFDM for Optical Communications (Elsevier/Academic Press, 2009).

[2] A. J. Lowery, L. B. Du, "Optical orthogonal division multiplexing for long haul optical communications: A review of the first five years," Optical Fiber Technology 17 (2011) 421-438.

[3] N. Cvijetic, "OFDM for Next-Generation Optical Access Networks," IEEE J. Lightw. Technol., vol. 30, no. 4, feb. 2012. 
[4] E. Wong, "Next-generation broadband access networks and technologies," J. Lightw. Technol., vol. 30, no. 4, pp. 597-608, Feb. $15,2012$.

[5] H. Ochiai and H. Imai, "On the Distribution of the Peak-to-Average Power Ratio in OFDM Signals," IEEE Trans. Commun., vol. 49, no. 2, Feb. 2001, pp. 282-89.

[6] N. A. Olsson, "Lightwave systems with optical amplifiers," J. Lightw. Technol., vol. 7, no. 7, pp. 1071-1082, Jul. 1989.

[7] D. R. Zimmerman, and L. H. Spiekman, "Amplifiers for the masses: EDFA, EDWA, and SOA amplets for metro and access networks," J. Lightwave Technol. 22(1), 63-70 (2004).

[8] G. Contestabile, Y. Yoshida, A. Maruta, and K. Kitayama, "Ultra-broad band, low power, highly efficient coherent wavelength conversion in quantum dot SOA," Optics Express, vol. 20, no. 25, pp. 27902-27907, 2012.

[9] H. Khaleghi, A. Sharaiha, T. Rampone, P. Morel, and M. Guégan, "Semiconductor Optical Amplifiers in Coherent Optical-OFDM Systems," IEEE Photon. Tech. Lett., vol. 24, no. 7, pp. 560-562, April 2012.

[10] H. Khaleghi, P. Morel, A. Sharaiha, and T. Rampone, "Experimental Validation of Numerical Simulations and Performance Analysis of a Coherent Optical-OFDM Transmission System Employing a Semiconductor Optical Amplifier," IEEE J. Lightwave Technol., vol. 31, no. 1, pp. 161-170, January 2013.

[11] M. J. Connelly, Semiconductor Optical Amplifiers. Boston, MA:Kluwer, 2002.

[12] T Jiang, Y Wu, "An overview: peak-to-average power ratio reduction techniques for OFDM signals," IEEE Trans. Broadcast., 2008.

[13] Y. Rahmatallah and S. Mohan, "Peak-to-average power ratio reduction in OFDM systems: A survey and taxonomy," IEEE Communications Surveys and Tutorials, accepted for publication, February 2013.

[14] B. Goebel, S. Hellerbrand, N. Haufe and N. Hanik, "PAPR reduction techniques for coherent optical OFDM transmission," in Proc. ICTON 2009, Jul. 2009.

[15] Y. London, D. Sadot, "Nonlinear effects mitigation in coherent optical OFDM system in presence of high peak power," J. Lightw. Technol., 29 (21) (2011).

[16] B. Liu, X. Xin, L. Zhang, J. Yu, "277.6-Gb/s LDPC-coded CO-OFDM transmission system with low PAPR based on subcarrier pre-filtering technology", Optics Communications, 285, pp. 5397-5400, 2012.

[17] Y. Tang, K. Ho, and W. Shieh, "Coherent optical OFDM transmitter design employing predistortion," IEEE Photon. Technol. Lett., vol. 20, no. 11, pp. 954-956, Jun. 2008.

[18] D. J. F. Barros and J. M. Kahn, "Optical modulator optimization for orthogonal frequency-division multiplexing," J. Lightwave Tech., vol. 27, pp. 2370-2378, Jul. 1, 2009.

[19] L. Chen, B. Krongold, and J. Evans, "Theoretical Characterization of Nonlinear Clipping Effects in IM/DD Optical OFDM Systems," IEEE Trans. Commun., vol. 60, No. 8, aug. 2012.

[20] Y. Hao, Y. Li, R. Wang, W. Huang, "Fiber nonlinearity mitigation by PAPR reduction in coherent optical OFDM systems via biased clipping OFDM," Chinese Optics Lett., Jan. 2012.

[21] C. Zhang, C. Chen, J. Huang, and K. Qiu, "Performance improvement of optical OFDMA-based PON using data clipping and additional phases," IEEE Photon. Technol. Lett. 24(4), 255-257 (2012).

[22] H. S. Chung, S. H. Chang, and K. J. Kim, "Companding Transform Based SPM Compensation in Coherent Optical OFDM Transmission," Opt. Express, vol. 16, no. 26, pp. 702- 709, dec. 2011.

[23] H. Chen, J. Yu, J. Xiao, Z. Cao, F. Li, L. Chen, "Nonlinear effect mitigation based on PAPR reduction using electronic predistortion technique in direct-detection optical OFDM system," Optical Fiber Technology, Vol. 19, Issue 5, , pp. 387-391, oct. 2013.

[24] J. Xiao, J. Yu, X. Li, Q. Tang, H. Chen, F. Li, Z. Cao, and L. Chen, "Hadamard transform combined with companding transform technique for PAPR reduction in an optical direct-detection OFDM system," IEEE J. Opt. Commun. Netw., 4(10), 709-714.

[25] X. B. Wang, T. T. Tjhung, and C. S. Ng, "Reduction of peak-to-average power ratio of OFDM system using a companding technique," IEEE Trans. Broadcast., vol. 45, no. 3, pp. 303-307, Sep. 1999.

[26] Y. Wang, J. Ge, L. Wang, J. Li, B. Ai, "Nonlinear Companding Transform for Reduction of Peak-to-Average Power Ratio in OFDM Systems," IEEE Trans. Broadcast., vol. 59, no. 2, june 2013.

[27] J. R. Barry and E. A. Lee, "Performance of Coherent Optical Receivers," Proceeding of The IEEE, Vol. 78, No. 8, August 1990, pp.1369-94.

[28] W. Shieh, H. Bao, Y. Tang, Coherent optical OFDM: theory and design, Optics Express, 2008.

[29] R. L. Haupt and S. E. Haupt, Practical Genetic Algorithms. New Jersey: Wiley- Interscience, 2004. 